\title{
Complicated Pulmonary Pseudocyst Following Traumatic Lung Injury Rescued by Extracorporeal Membrane Oxygenation
}

Sung Bum Park, M.D. ${ }^{1}$, Dae Sang Lee, M.D. ${ }^{1}$, Jeong-Am Ryu, M.D. ${ }^{1}$, Jong Ho Cho, M.D. ${ }^{2}$, Yang Hyun Cho, M.D. ${ }^{2}$, Chi Ryang Chung, M.D. ${ }^{1}$, Jeong Hoon Yang, M.D., Ph.D. ${ }^{1,3}$, Kyeongman Jeon, M.D., Ph.D. ${ }^{1,3}$, Gee Young Suh, M.D., Ph.D. ${ }^{1,3}$, and Chi-Min Park, M.D., Ph.D. ${ }^{1,4}$

Departments of ${ }^{1}$ Critical Care Medicine, ${ }^{2}$ Thoracic and Cardiovascular Surgery, ${ }^{3}$ Internal Medicine, and ${ }^{4}$ Surgery, Samsung Medical Center, Sungkyunkwan University School of Medicine, Seoul, Korea

Traumatic pulmonary pseudocyst is a rare complication of blunt chest trauma that usually appears immediately in children or young adults and is characterized by a single or multiple pulmonary cystic lesions on chest radiography and has spontaneous resolution of the radiologic manifestations. However, we experienced a case of a delayed complicated pulmonary pseudocyst in a 17-year-old boy following severe traumatic acute respiratory distress syndrome rescued by Veno-venous extracorporeal membrane oxygenation (ECMO). In this case, the pseudocyst appeared on the 12th day after trauma and transformed into an infected cyst. Veno-venous ECMO was successfully maintained for 20 days without anticoagulation.

Key Words: acute respiratory distress syndrome; extracorporeal membrane oxygenation; traumatic pulmonary pseudocyst.

Traumatic pulmonary pseudocyst, also referred to as traumatic pneumatocele or lung cyst, is a rare complication of blunt chest trauma. The most common clinical manifestation of lung parenchymal injury after blunt chest trauma is pulmonary contusion.[1] However, parenchymal lacerations can also occur in children or young adults and can result in cavities referred to as traumatic pulmonary pseudocyst.[2] Traumatic pulmonary pseudocyst is trapped air within a lacerated lung parenchyma, so it appears as a thin-walled, air-filled cavity on chest radiography. Single or multiple pulmonary pseudocyst can be found in any location but rarely occur at the apices.[3] Since such pseudocysts are acutely formed primary structures that appears shortly after blunt chest trauma

Received on March 10, 2014 Revised on May 9, 2014

Accepted on May 9, 2014

Correspondence to: Chi-Min Park, Departments of Critical Care Medicine and Surgery, Samsung Medical Center, Sungkyunkwan University School of Medicine, 81 Irwon-ro, Gangnam-gu, Seoul 135-710, Korea

Tel: +82-2-3410-1096, Fax: +82-2-3410-6980

E-mail:dr99.park@samsung.com

* No potential conflict of interest relevant to this article was reported. and have a self-limited course, invasive intervention is usually unnecessary in patients with closed chest trauma.[4] We present the case of a 17-year-old boy with an unusual form of pulmonary pseudocyst following severe traumatic lung injury who was rescued by anticoagulation-free veno-venous extracorporeal membrane oxygenation (ECMO).

\section{Case Report}

A 17-year-old boy was injured when his motorcycle apparently crashed into the side of a bus. He was found under the bus unconscious when emergency services arrived. He presented to our emergency department (ED) within 20 minutes. At the initial physical examination, he presented with stupor, blood pressure of 134/106 mmHg, tachycardia over 145 beats per minute, tachypnea over 30 breaths per minute, and hypoxic respiratory failure with $76 \%$ oxygen saturation on room air. There were several abrasions with bruises on his face and right chest wall. His breathing sounds were decreased on the right side. His abdomen was soft without tenderness but showed mild distension. His ex- 

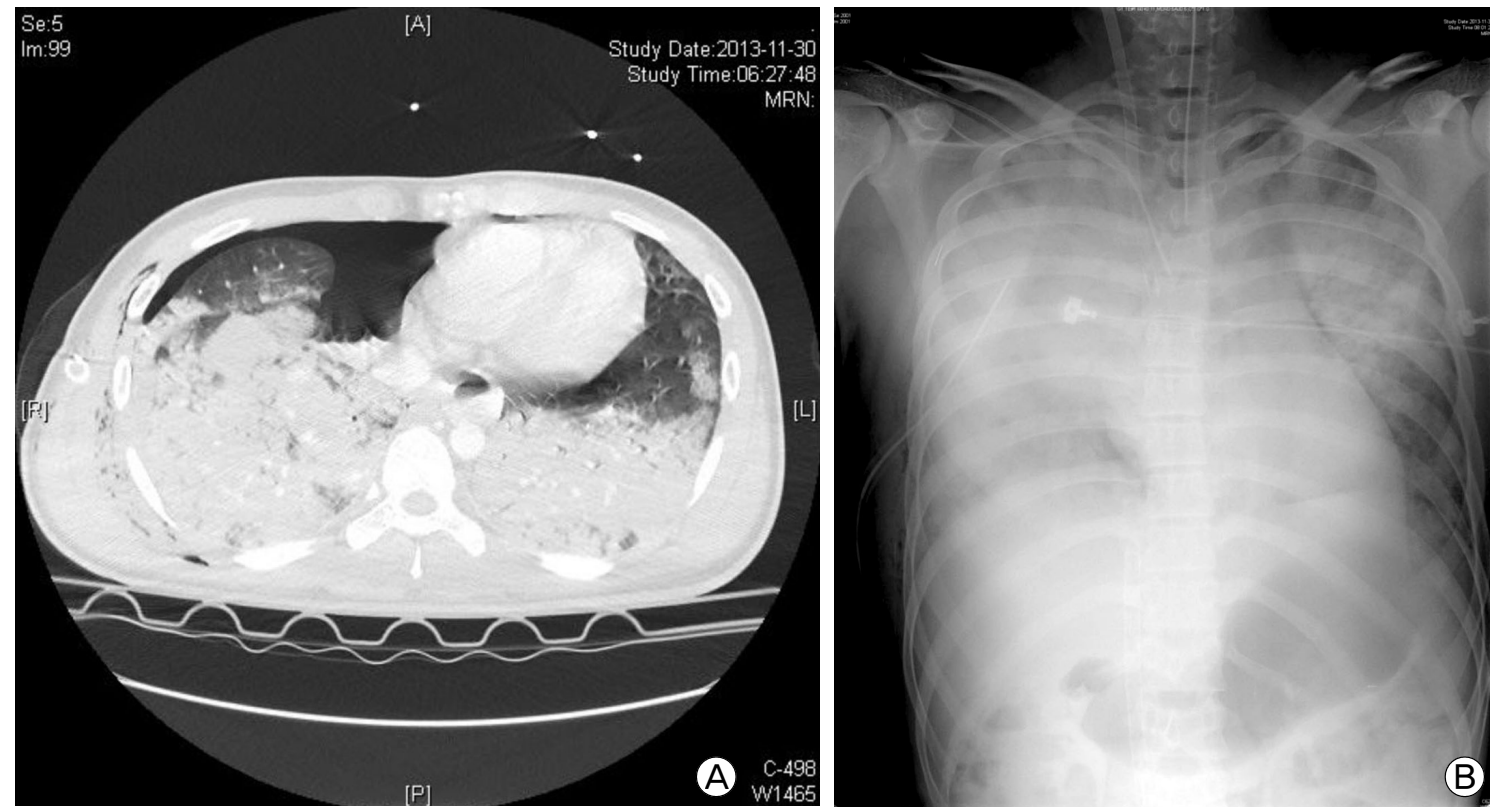

Fig. 1. Initial chest radiography after trauma. (A) Traumatic pneumothorax with a bilateral multiple lung contusion. (B) Rapidly progressing traumatic ARDS.
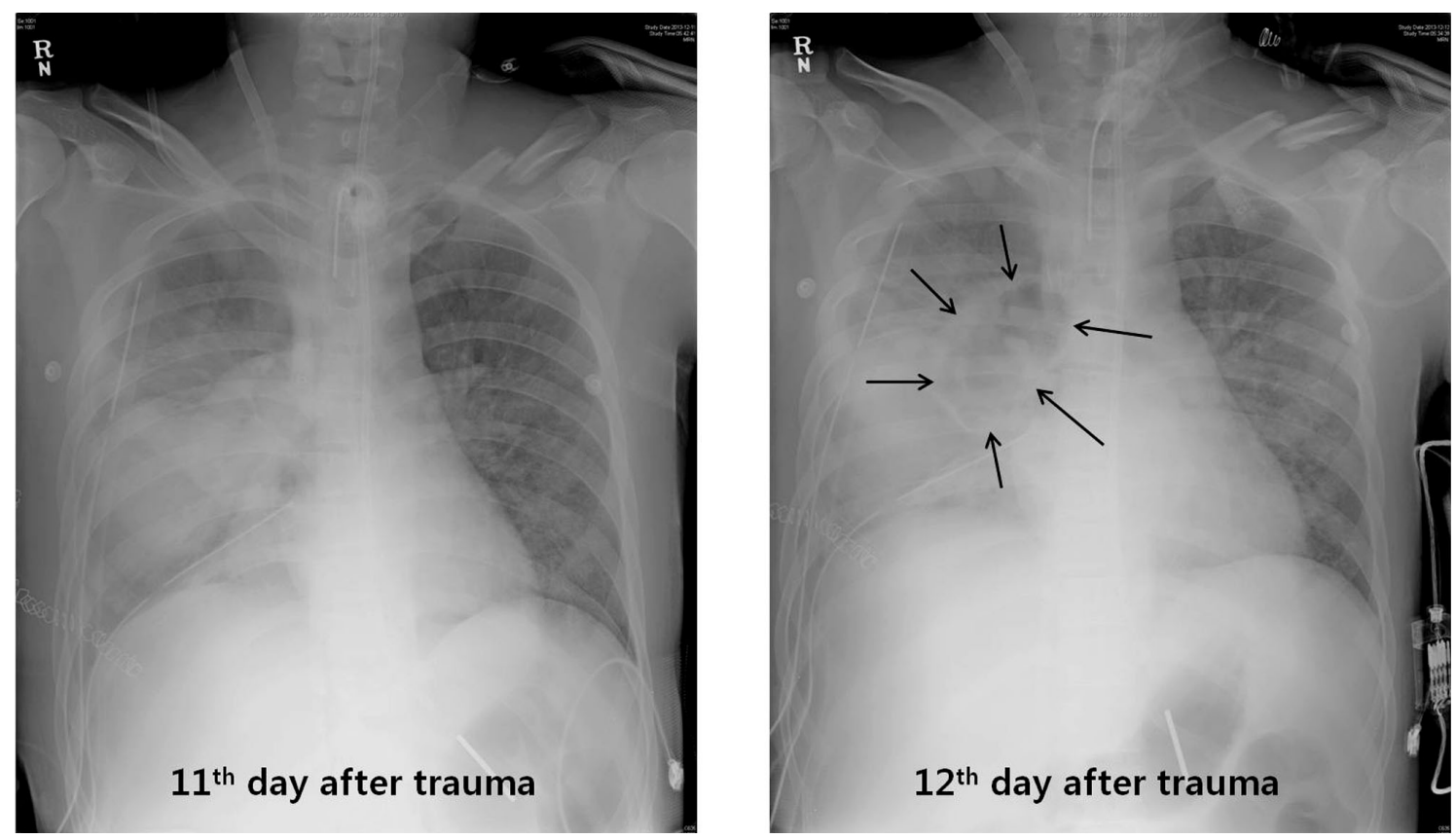

Fig. 2. Newly appeared cavitary lesion at the previous contusion site on the 12th day after trauma (black arrows).

tremities were intact, except for the left arm.

Chest radiography and CT scan showed right pneumothorax with multiple pulmonary contusions, but there was no rib fracture or cavitary lesion in the lung parenchyma (Fig. 1A). Abdominal CT scan showed lower abdominal hemoperitonium related to a grade IV urethral injury as per the urethral injury scale and fractures of the sacrum and bilateral pubic ramus. A fracture of the body of the left humerus was found in radiography of the extremities. Taken together, the injury severity score was 29 . Adequate therapeutic oxygen supply was administered, but it did not resolve hypoxia, so mechanical ventilatory support was applied. A chest tube was inserted into right pleural space for management of pneumothorax, and $1400 \mathrm{cc}$ of bloody pleural effusion was drained over 3.5 hours. A pelvic binder was applied 
for management of the pelvic bone fracture. Nonetheless, type I and type II respiratory failure rapidly exacerbated, and the patient experienced severe acute respiratory distress syndrome (ARDS) exceeding the maximal capability of the mechanical ventilator (Fig. 1B). As a result, we applied veno-venous ECMO for ARDS 3 hours after ED arrival but did not use any medication of anticoagulation because of intra-abdominal and intra-thoracic bleeding.

We initially set up a ventilator at fraction of inspired oxygen $\left(\mathrm{FiO}_{2}\right)$ of 0.4 , positive end-expiratory pressure (PEEP) of 10 $\mathrm{cmH}_{2} \mathrm{O}$, pressure above PEEP of $10 \mathrm{cmH}_{2} \mathrm{O}$ (predictive body weight $70.57 \mathrm{~kg}$ ), peak pressure of $20 \mathrm{cmH}_{2} \mathrm{O}$, respiratory rate of 10 breaths per minute in pressure control mode with deep seda- tion, with the aim of lung-protective ventilation strategy for ARDS management. Veno-venous ECMO was set up at $\mathrm{FiO}_{2}$ of 1.0, sweep gas flow of $8.0 \mathrm{~L} / \mathrm{min}$, and blood flow of $4.0 \mathrm{~L} / \mathrm{min}$ to maintain proper arterial gaseous status. However, chest radiography showed haziness of both lungs, and tidal volume did not exceed $30 \mathrm{ml}$ for four days. On the fifth day after trauma, lungs began to show radiographic improvement, and tidal volume also neared $150 \mathrm{ml}$ at the initial ventilator settings given above. However, we continued to maintain the initial settings of the ventilator and ECMO device in order to provide lung rest. On the 17 th day after trauma, since the tidal volume exceeded $360 \mathrm{ml}$ at the same ventilator settings, we increased the pressure above
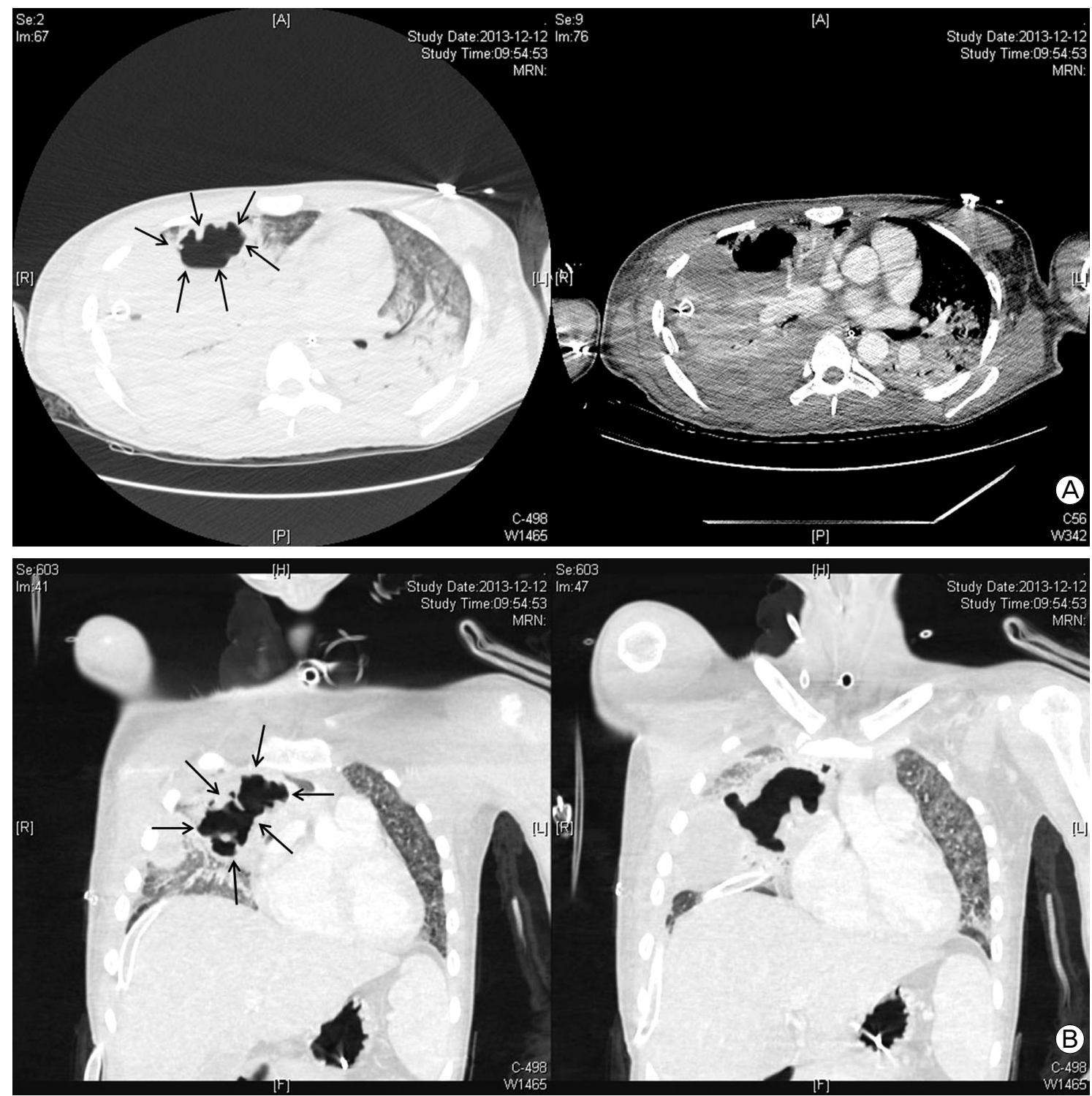

Fig. 3. Delayed large traumatic pulmonary pseudocyst. Delayed laceration of previous contused lung parenchyma made a single pulmonary pseudocyst. (black arrows) (A) Axial view. (B) Coronal view. 
PEEP but lowered the PEEP and sweep gas flow of ECMO.

With regard to ECMO management, since the patient had severe bleeding from his chest tube and urinary catheter, we were not able to initiate any anticoagulation. To prevent thrombotic complications of ECMO, we regularly checked serum lactate dehydrogenase, plasma hemoglobin, and pressures across the oxygenator. We did not experience any serious complications from off-anticoagulation. ECMO was successfully maintained and removed on the 20th day after trauma. The patient was able to breathe unassisted five days after ECMO weaning.
On the 12th day after trauma, a new cavitary lesion appeared abruptly at the previous contusion site on the right lung on the daily follow-up chest radiography (Fig. 2). The large single cavitary lesion, $6.6 \mathrm{~cm}$ in height, $7.8 \mathrm{~cm}$ in width, and with a $3.2 \mathrm{~cm}$ antero-posterior diameter was characterized as a thin-walled, air-filled cavity on chest CT scan (Fig. 3). Since we assessed the cavity as a traumatic pulmonary pseudocyst appearing by delayed laceration of previously contused lung parenchyma, we observed the cavity without any intervention or invasive diagnostic examination, expecting a typical self-limited course of the disease.
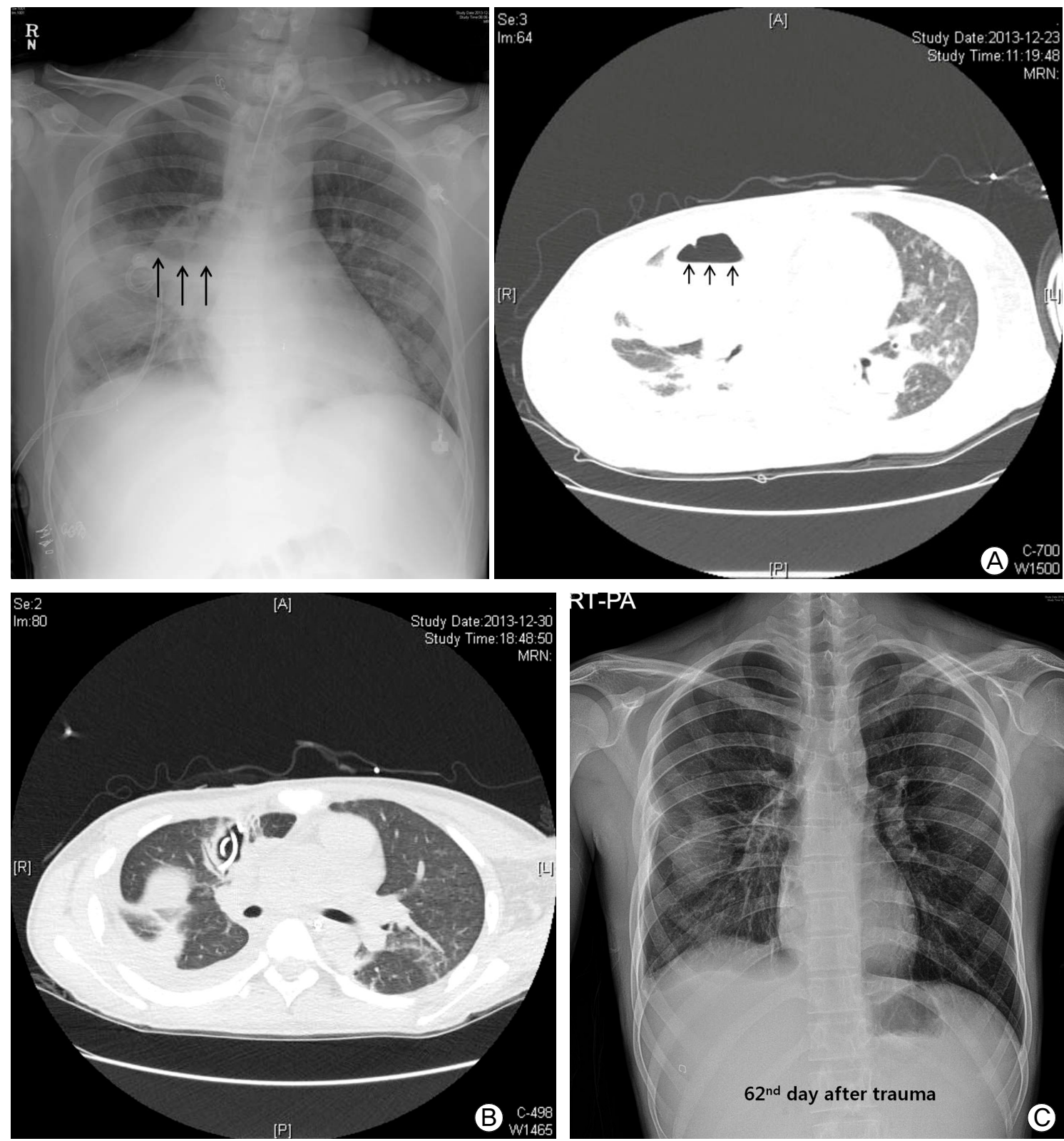

Fig. 4. Hospital course of the pseudocyst. (A) Complicated pulmonary pseudocyst showing air-fluid level. (B) Resolving pseudocyst after percutaneous drainage. (C) Complete resolution of pseudocyst. 
However, fever developed and increased intra-cavitary fluid was revealed on chest radiography on the 23rd day after trauma (Fig. $4 \mathrm{~A}$ ), so we inserted a percutaneous drain catheter into the cavity and drained pus-like fluid. Finally, once fever subsided, the amount of drainage decreased to nearly $0 \mathrm{ml}$ per day, and follow-up CT scan showed decreased size of the cavity (Fig. 4B), the drainage catheter was successfully removed without any complication on the eighth day after insertion.

Finally, the patient was transferred to the general ward on the 37 th day after trauma and was discharged after 36 days of rehabilitation in the general ward. Furthermore, the previous cavitary lesion disappeared on the chest radiography at discharge (Fig. 4C).

\section{Discussion}

The most common complications of closed blunt chest trauma are pulmonary contusion and intra-thoracic hematoma. Traumatic pulmonary pseudocyst rarely develops, accounting for only $3 \%$ of overall lung parenchymal injury.[5] Traumatic pulmonary pseudocyst generally occurs in children or young adults as a result of parenchymal laceration. Traumatic pulmonary pseudocyst usually appears on chest radiography as a round or ovoid cyst containing air or fluid surrounded by thin wall lining and usually appears shortly after blunt chest trauma.[4,5] It is usually detected within 12 hours following trauma but can rarely appear several days later.[4] Considering that delayed traumatic pulmonary pseudocyst appears at lung contusion sites suggests that contused lung parenchyma is vulnerable and has a risk of delayed laceration resulting in pseudocyst in younger patients.

The detailed mechanism of its pathology remains uncertain. However, it is presently widely accepted that traumatic pulmonary pseudocyst develops when a shearing force penetrates the vulnerable thoracic cage, and that a two-step mechanism is involved in this development.[5-8] The first step is trauma-induced rapid compression of lung parenchyma, which results in barotrauma, bursting, and rupture of the lung parenchyma. The second step is decompression with elastic recoil of the same injured site. This produces a localized increase in negative intra-thoracic pressure at the injured site and results in laceration of parenchyma, leading to formation of a cavitary lesion. In terms of disease prevalence, the majority of patients are children or adults younger than 30 years. It seems that younger people have more vulnerable and pliable thoracic cage than older adults; thus, when external kinetic energy impacts the thoracic cage, it more easily penetrates the lung parenchyma and imparts a shearing force resulting in laceration.[8-10]

The common clinical manifestations of these occurrences are atypical chest pain, dyspnea, tachypnea, cough, and blood-tinged sputum but the symptoms are often subtle and not specific. $[5,11]$ Traumatic pulmonary pseudocyst usually has a self-limited benign course, and clinical observation without any intervention is generally successful in most cases. However, there have been a few cases of severe complications, such as gross hemoptysis, infection with abscess formation, secondary hematocele, respiratory deterioration, and failure to resolve.[12] These severe complications usually develop in cases of large pseudocyst greater than $4 \mathrm{~cm}$ in diameter.[12-14]

ECMO is not widely used in traumatic patients because of the increased risk of bleeding. However, in our experience, veno-venous ECMO can be safely maintained without anticoagulation in bleeding condition, although the durability of the centrifugal pump and oxygenator will decrease and the risk of bleeding may also be increased even without anticoagulation. However, the bleeding should be controlled before ECMO or controllable after stabilization by ECMO.

In conclusion, we experienced a rare case of delayed complicated traumatic pulmonary pseudocyst in a patient with closed chest and abdominal trauma. Furthermore, ECMO can be a viable option in trauma patients with controllable bleeding.

\section{References}

1) Fulton RL, Peter ET: The progressive nature of pulmonary contusion. Surgery 1970; 67: 499-506.

2) Blane CE, White SJ, Wesley JR, Coran AG: Immediate traumatic pulmonary pseudocyst formation in children. Surgery 1981; 90: 872-5.

3) Galea MH, Williams N, Mayell MJ: Traumatic pneumatocele. J Pediatr Surg 1992; 27: 1523-4.

4) Athanassiadi K, Gerazounis M, Kalantzi N, Kazakidis P, Fakou A, Kourousis D: Primary traumatic pulmonary pseudocysts: a rare entity. Eur J Cardiothorac Surg 2003; 23: 43-5.

5) Kato R, Horinouchi H, Maenaka Y: Traumatic pulmonary pseudocyst. Report of twelve cases. J Thorac Cardiovasc Surg 1989; 97: 309-12.

6) Fagan CJ, Swischuk LE: Traumatic lung and paramediastinal pneumatoceles. Radiology 1976; 120: 11-8.

7) Khan FA, Phillips W, Khan A, Seriff NS: Unusual unilateral 
blunt chest trauma without rib fractures leading to pulmonary laceration requiring pneumonectomy. Chest 1974; 66: 211-4.

8) Steinhausen E, Bouillon B, Yücel N, Tjardes T, Rixen D, Paffrath T, et al: Nonoperative management of post-traumatic pulmonary pseudocyst after severe thoracic trauma and hemorrhage by coagulation management, kinetic therapy, and control of secondary infection: a case report. J Trauma 2007; 63: 1391-4.

9) Crestanello JA, Samuels LE, Kaufman MS, Thomas MP, Talucci R: Posttraumatic pulmonary pseudocyst. J Trauma 1998; 44: 401-3.

10) Romero J, Velmahos GC: Giant posttraumatic hemopneumatocele: nonoperative management is safe. J Trauma 2000; 49: 1132-4.
11) Ganske JG, Dennis DL, Vanderveer JB Jr: Traumatic lung cyst: case report and literature review. J Trauma 1981; 21: 493-6.

12) Chon SH, Lee CB, Kim H, Chung WS, Kim YH: Diagnosis and prognosis of traumatic pulmonary psuedocysts: a review of 12 cases. Eur J Cardiothorac Surg 2006; 29: 819-23.

13) Melloni G, Cremona G, Ciriaco P, Pansera M, Carretta A, Negri G, et al: Diagnosis and treatment of traumatic pulmonary pseudocysts. J Trauma 2003; 54: 737-43.

14) Van Hoorebeke E, Jorens PG, Wojciechowski M, Salgado R, Desager K, Van Schil P, et al: An unusual case of traumatic pneumatocele in a nine-year-old girl: a bronchial tear with clear bronchial laceration. Pediatr Pulmonol 2009; 44: 826-8. 